\title{
ON CHANGING FIXED POINTS AND COINCIDENCES TO ROOTS
}

\author{
ROBIN BROOKS AND PETER WONG \\ (Communicated by Frederick R. Cohen)
}

\begin{abstract}
The coincidence problem, finding solutions to $f(x)=g(x)$, can sometimes be converted to a root problem, finding solutions to $\sigma(x)=a$. As an application, we show that for any two maps $f, g: M \rightarrow M, N(f, g)=$ $|L(f, g)|$ where $M$ is a compact connected nilmanifold, $N(f, g)$ and $L(f, g)$ are the Nielsen and Lefschetz numbers, respectively, of $f$ and $g$. This result in the case where $g$ is the identity is due to D. Anosov.
\end{abstract}

\section{INTRODUCTION}

Let $G$ be a compact connected Lie group and $K$ be a closed subgroup. Denote by $M=G / K$ the homogeneous space of right cosets. In [7] Fadell observed that every selfmap $f: M \rightarrow M$ has a fixed point if and only if there is a solution to the root problem $\psi(g)=e K \quad(e \in G$ the unit) for every $K$-map $\psi: G \rightarrow M$. Here $K$ acts on $G$ via $k \circ g=g k^{-1}$ and $K$ acts on $M$ via $k \circ g K=k g K$. The root problem is often easier to analyze, solve [2-4, 11] in particular, because root classes of maps into closed orientable manifolds always have the same index. Accordingly, we convert the fixed point and coincidence problems for maps of nilmanifolds into a root problem and generalize to coincidences (Theorem 3.3) Anosov's result [1] that for any selfmap $f: M \rightarrow M$ on a compact nilmanifold $M$, the Nielsen fixed point classes of $f$ have the same index each of which is $0,+1$, or -1 .

Throughout $H_{*}$ and $H^{*}$ will denote singular homology and cohomology with integer coefficients, respectively.

During the preparation of the manuscript, we learned that Theorem 3.3 was also obtained by Jezierski [9] and McCord [12] using different methods. We thank Bob Brown for bringing [9] to our attention and Chris McCord for his preprint.

We also thank the referee for a number of helpful suggestions.

\section{LOCAL NIELSEN ROOT THEORY}

In this section we introduce a local version of the root theory as in [2] (see also $[3,4,11])$, which we need in $\S 3$.

Received by the editors May 8, 1990.

1991 Mathematics Subject Classification. Primary 55M20.

Key words and phrases. Fixed points, coincidences, roots, Lefschetz number, Nielsen number, degree, nilmanifold. 
Let $X$ and $Y$ be oriented $n$-manifolds and $e \in Y$. Suppose that $f: U \rightarrow Y$ is a map defined on an open subset $U \subset X$ such that the set of roots $R_{f}=$ $\{x \in U \mid f(x)=e\}$ is compact in $U$. Define the root index of $f$ in $U$ to be the integer

$$
\omega(f ; U)=\left\langle f^{*} \mu_{Y}, o_{R_{f}}\right\rangle
$$

where $o_{R_{f}} \in H_{n}\left(U, U-R_{f}\right)$ is the fundamental homology class around $R_{f}$, $\mu_{Y} \in H^{n}(Y, Y-e)$ is the preferred generator, and $f^{*}: H^{n}(Y, Y-e) \rightarrow$ $H^{n}\left(U, U-R_{f}\right)$.

Proposition 2.1. (1) (Homotopy) If $F: U \times I \rightarrow Y$ is a homotopy such that $K=\bigcup_{t} R_{F_{t}}$ is compact in $U$ then

$$
\omega\left(F_{0} ; U\right)=\omega\left(F_{1} ; U\right) .
$$

(2) (Additivity) If $U=\bigsqcup_{i=1}^{k} U_{i}$ and $R_{f \mid U_{i}}$ is compact in $U_{i}$ for each $i$, then

$$
\omega(f ; U)=\sum_{i=1}^{k} \omega\left(f \mid U_{i} ; U_{i}\right) .
$$

Proof. (i) For each $t, 0 \leq t \leq 1$, the inclusion $j:(U, U-K) \hookrightarrow\left(U, U-R_{F_{t}}\right)$ induces $j_{*}$ that takes $o_{K}$ to $o_{R_{F_{t}}}$. Since

$$
\left\{F_{t}\right\}:(U, U-K) \rightarrow(Y, Y-e)
$$

is a homotopy, it follows that

$$
\left\langle j^{*} F_{0}^{*} \mu_{Y}, o_{K}\right\rangle=\left\langle j^{*} F_{1}^{*} \mu_{Y}, o_{K}\right\rangle .
$$

Thus,

and hence,

$$
\left\langle F_{0}^{*} \mu_{Y}, j_{*} o_{K}\right\rangle=\left\langle F_{1}^{*} \mu_{Y}, j_{*} o_{K}\right\rangle
$$

$$
\omega\left(F_{0} ; U\right)=\left\langle F_{0}^{*} \mu_{Y}, o_{R_{F_{0}}}\right\rangle=\left\langle F_{1}^{*} \mu_{Y}, o_{R_{F_{1}}}\right\rangle=\omega\left(F_{1} ; U\right) .
$$

(ii) Since $R_{f}=\bigsqcup_{i=1}^{k} R_{f \mid U_{i}}$, it follows that

$$
\left\langle f^{*} \mu_{Y}, o_{R_{f}}\right\rangle=\sum_{i=1}^{k}\left\langle\left(f \mid U_{i}\right)^{*} \mu_{Y}, o_{R_{f} \mid U_{i}}\right\rangle .
$$

Two roots $x, y \in R_{f}$ are said to be Nielsen equivalent if there is a path $\alpha:[0,1] \rightarrow U$ such that $\alpha(0)=x, \alpha(1)=y$, and $f \circ \alpha \sim e$ (rel. end points). It is easy to verify that this is an equivalence relation on $R_{f}$ and the equivalence classes are called root classes of $f$. Denote by $\Gamma(f, U)$ the set of root classes of $f$.

Proposition 2.2. Each root class is open in $R_{f}$ and hence the set $\Gamma(f, U)$ is finite.

Proof. The first assertion follows from [11, V.3.3] and the second is then obvious since $R_{f}$ is compact in $U$.

Given a root class $\alpha \in \Gamma(f, U)$, choose an open neighborhood $V$ of $\alpha$ in $U$ such that $V \cap R_{f}=\alpha$. Then the root index of the class $\alpha$ is given by

$$
\omega(f ; \alpha):=\omega(f \mid V ; V) \text {. }
$$

Note that $\omega(f ; \alpha)$ is defined independent of the choice of $V$. A root class $\alpha$ is said to be essential if $\omega(f ; \alpha) \neq 0$.

We now prove a local version of $[2$, Corollary 3$]$ (or [11, V.7.1]). 
Theorem 2.3. ${ }^{1}$ Let $U$ be a connected open subset of an oriented n-manifold $X$. Let $f: U \rightarrow Y$ be a map where $Y$ is an oriented triangulated $n$-manifold with $e \in Y$. Suppose that $R_{f}$ is compact and $\alpha$ is an essential root class of $f$. Then for any root class $\beta \in \Gamma(f, U), \omega(f ; \alpha)=\omega(f ; \beta)$.

Proof. Since $R_{f}$ is compact in $U$, there exists a compact connected subset $K$ such that $R_{f} \subset$ int $K \subset K \subset U$.

Suppose that $\beta \in \Gamma(f, U)-\{\alpha\}$. Let $x \in \alpha$ and $y \in \beta$. Choose a path $C$ in $K$ with $C(0)=x, C(1)=y$ and a regular neighborhood $V$ of the loop $f \circ C$ in $Y$ such that $f^{-1}(V) \subset$ int $K$. Since $Y$ is a manifold, there exists an isotopy $H: Y \times I \rightarrow Y$ such that $H_{1}=1_{Y}, H_{t}(e)=f \circ C(t)$, and $H_{t}(y)=y$ for all $y \in Y-V$. Define $F: U \times I \rightarrow Y$ by $F_{t}=H_{t} \circ f$. Then

$$
\begin{aligned}
\left\{F_{t}(C(1-t))\right\} & =\left\{H_{t} \circ f \circ C(1-t)\right\} \\
& =\left\{H_{t} \circ H_{1-t}(e)\right\} \sim\{e\} \quad \text { (rel. end points). }
\end{aligned}
$$

Thus $x$ and $y$ are Nielsen equivalent as roots of $F$ in $U \times I$. Hence $\alpha$ and $\beta$ belong to the same root class $\mathcal{N}$ of $F$ for some $\mathscr{N} \in \Gamma(F, U \times I)$. Let $\mathscr{N}_{t}=p(\mathscr{N} \cap(U \times\{t\}))$ where $p(x, s)=x, s \in I$. Note that $\alpha=\mathscr{N}_{1}$ and $\beta=\mathscr{N}_{0}$. Since $H_{t}$ is the identity map outside $V$, the set of roots $R_{F}$ of $F$ is compact. Choose an open neighborhood $W$ of $\mathscr{N}$ in $U \times I$ so that $\operatorname{cl}(W)$ does not contain any other roots of $F$. For any $r, 0 \leq r \leq 1, K_{r}=p\left(R_{F}\right)-W_{r}$ is compact in $U$ where $W_{r}=p(W \cap(U \times\{r\}))$. By uniform continuity, for any $\varepsilon>0$, there exists $\delta>0$ such that $|r-s|<\delta$ implies $d\left(F_{r}(x), F_{s}(x)\right)<\varepsilon$ for all $x \in K_{r}$. This implies that $F_{s}$ has no roots in $K_{r}$ and hence $\mathscr{N}_{s} \subset W_{r}$. Define $\left\{\Gamma_{t}^{r, s}\right\}: U \rightarrow Y$ by

$$
\Gamma^{r, s}(x, t)=F(x,(1-t) r+t s)
$$

Then

$$
\Gamma_{0}^{r, s}=F_{r}, \quad \Gamma_{1}^{r, s}=F_{s}
$$

and $\bigcup_{t} R_{\Gamma_{t}^{r}}, \cap W_{r}$ is compact in $W_{r}$. By 2.1(1), we have

$$
\omega\left(F_{r} ; \mathscr{N}_{r}\right)=\omega\left(\Gamma_{0}^{r, s} \mid W_{r} ; W_{r}\right)=\omega\left(\Gamma_{1}^{r}, s \mid W_{r} ; W_{r}\right)=\omega\left(F_{s} ; \mathscr{N}_{s}\right) .
$$

By compactness of $I$, we conclude that

$$
\omega\left(F_{0} ; \beta\right)=\omega\left(F_{0} ; \mathscr{N}_{0}\right)=\omega\left(F_{1} ; \mathscr{N}_{1}\right)=\omega\left(F_{1} ; \alpha\right) ;
$$

therefore,

$$
\omega\left(H_{0} \circ f ; \beta\right)=\omega(f ; \alpha) .
$$

Since $H_{0}$ is isotopic to $H_{1}=1_{Y}$ and $Y$ is orientable, $\omega\left(H_{0} \circ f ; \beta\right)=\omega(f ; \beta)$ and thus the proof is complete.

\section{ANOSOV'S THEOREM FOR COINCIDENCES}

Recall that a compact connected nilmanifold $M$ is a homogeneous space of the form $G / \Gamma$ where $G$ is a connected and simply connected nilpotent Lie group and $\Gamma$ is a uniform discrete subgroup. For any two maps $f, g: M \rightarrow M$,

\footnotetext{
${ }^{1}$ After the authors sent proofs to the editor, Professor Boju Jiang pointed out that Theorem 2.3 is incorrect. The main Theorem 3.3, however, remains valid provided an alternate argument replaces the role that Theorem 2.3 played in Lemma 3.1. This argument will appear in a subsequent correction.
} 
we let $C_{f, g}=\{x \in M \mid f(x)=g(x)\}$ be the set of coincidences of $f$ and $g$. As in [4], two coincidences $x, y \in C_{f, g}$ are Nielsen equivalent as coincidences if there is a path $\alpha$ in $M$ from $x$ to $y$ such that $f \circ \alpha \sim g \circ \alpha$ (rel. end points). We also have the notion of coincidence index and coincidence classes. The Lefschetz number $L(f, g)$ and the Nielsen number $N(f, g)$ are defined (see $[4,13,15])$. In this section, we will show that $N(f, g)=|L(f, g)|$, which generalizes a result of Anosov [1] in which case $g$ is the identity (see also [8]).

Lemma 3.1. Let $M$ be a connected compact nilmanifold of $\operatorname{dim} M \geq 3$. For any two maps $f_{1}, f_{2}: M \rightarrow M$, each coincidence class has the same index.

Proof. Write $M=G / \Gamma$ where $G$ is connected, simply connected, nilpotent and $\Gamma$ is a uniform discrete subgroup. By a result of Schirmer [14], we may assume without loss of generality that either $C_{f_{1}, f_{2}}=\varnothing$ or all the coincidence classes are essential, each of which contains only a single point. If $C_{f_{1}, f_{2}}=\varnothing$, the assertion is obvious.

Now let $g_{1}, \ldots, g_{k}$ be in $G$ such that $C_{f_{1}, f_{2}}=\left\{g_{1} \Gamma, \ldots, g_{k} \Gamma\right\}$. We can find $\Gamma$-maps $\psi_{1}, \psi_{2}: G \rightarrow M$ where $\psi_{i}(g)=g^{-1} f_{i}(g \Gamma), i=1,2$. Let $\tilde{g}=g_{j}$ for some $j, 1 \leq j \leq k$. Thus $\tilde{g} \Gamma \in C_{f_{1}, f_{2}}$. Choose lifts $\tilde{\psi}_{i}: G \rightarrow G$ of $\psi_{i}$, $i=1,2$ such that $\tilde{\psi}_{1}(\tilde{g})=\tilde{\psi}_{2}(\tilde{g})$. Let $U$ be an open $n$-disk containing $\left\{g_{1}, \ldots, g_{k}\right\}$ such that $C_{f_{1}, f_{2}} \subset p(U)$ where $p: G \rightarrow M$ is the projection. Define $\varphi: U \rightarrow M$ by

$$
\varphi(g)=\left(\tilde{\psi}_{1}(g)\right)^{-1} \tilde{\psi}_{2}(g) \Gamma .
$$

Then

$$
\varphi(g)=e \Gamma \Leftrightarrow f_{1}(g \Gamma)=f_{2}(g \Gamma)
$$

where $e$ is the unit in $G$. Thus the set of roots of $\varphi$ is given by $R_{\varphi}=$ $\left\{g_{1}, \ldots, g_{k}\right\}$.

Suppose that $g_{i}, g_{j} \in R_{\varphi}, i \neq j$ are Nielsen equivalent as roots of $\varphi$, i.e., there is a path $\alpha$ in $U$ from $g_{i}$ to $g_{j}$ and a homotopy (rel. end points) $H: I \times I \rightarrow M$ such that $H_{0}=\varphi \circ \alpha$ and $H_{1}=e \Gamma$. Define

$$
\widehat{H}(s, t)=\alpha(s) \tilde{\psi}_{1}(\alpha(s)) H(s, t) .
$$

Then

$$
\widehat{H}(s, 0)=\alpha(s) \tilde{\psi}_{1}(\alpha(s)) \varphi(\alpha(s))=f_{2}(\alpha(s) \Gamma)
$$

and, similarly, $\widehat{H}(s, 1)=f_{1}(\alpha(s) \Gamma)$. Moreover,

$$
\widehat{H}(0, t)=f_{1}\left(g_{i} \Gamma\right)=f_{2}\left(g_{i} \Gamma\right)
$$

and

$$
\widehat{H}(1, t)=f_{1}\left(g_{j} \Gamma\right)=f_{2}\left(g_{j} \Gamma\right) .
$$

This implies that $g_{i} \Gamma$ and $g_{j} \Gamma$ are Nielsen equivalent as coincidences of $f_{1}$ and $f_{2}$, contradicting the assumption that $\left\{g_{1} \Gamma, \ldots, g_{k} \Gamma\right\}$ are distinct classes. Now $g_{1}, \ldots, g_{k}$ are distinct root classes of $\varphi$ and hence, by Theorem 2.3, $\omega\left(\varphi ; g_{i}\right)=\omega\left(\varphi ; g_{j}\right)$ for all $i, j=1, \ldots, k$.

Note that the map $F_{i}(g)=g \tilde{\psi}_{i}(g)$ is a lift of $f_{i}$ for each $i=1,2$ and $F_{1}(\tilde{g})=F_{2}(\tilde{g})$. Choose a euclidean neighborhood $\widetilde{U}$ of $\tilde{g}$ containing no other roots of $\varphi$ and such that $p \mid \widetilde{U}$ is a homeomorphism. Consider the following 
commutative diagram

$$
\begin{aligned}
& \widetilde{U}, \widetilde{U}-\tilde{g} \quad \stackrel{\left(F_{1}, F_{2}\right)}{\longrightarrow} G \times G, G \times G-(p \times p)^{-1}(\Delta M) \stackrel{!}{\leftarrow} G, G-\Gamma \\
& p \mid \widetilde{U} \downarrow \\
& p(\tilde{U}), p(\tilde{U})-\tilde{g} \Gamma \stackrel{\left(f_{1}, f_{2}\right)}{\longrightarrow} \quad M \times M, M \times M-\Delta M \quad \stackrel{k}{\leftarrow} M, M-e \Gamma
\end{aligned}
$$

where $l(g)=(e, g)$ and $k(g \Gamma)=(e \Gamma, g \Gamma)$. Let $\sigma: G \times G, G \times G-$ $(p \times p)^{-1}(\Delta M) \rightarrow G, G-\Gamma$ be given by $\sigma\left(g, g^{\prime}\right)=g^{-1} g^{\prime}$.

Since $\left(F_{1}(g)\right)^{-1} F_{2}(g)=\left(\tilde{\psi}_{1}(g)\right)^{-1} \tilde{\psi}_{2}(g)$, it follows that

$$
k \circ p \circ \sigma \circ\left(F_{1}, F_{2}\right)=k \circ \varphi \text {. }
$$

Thus,

$$
\begin{aligned}
\varphi^{*} \circ k^{*} & =\left(F_{1}, F_{2}\right)^{*} \circ \sigma^{*} \circ p^{*} \circ k^{*} \\
& =\left(F_{1}, F_{2}\right)^{*} \circ(p \times p)^{*} \quad\left(\text { since } l^{*} \cong, \sigma^{*}=\left(l^{*}\right)^{-1}\right) \\
& =(p \mid \widetilde{U})^{*} \circ\left(f_{1}, f_{2}\right)^{*} .
\end{aligned}
$$

We have

$$
\left\langle\varphi^{*} \circ k^{*}\left(\nu_{M}\right), o_{\tilde{g}}\right\rangle=\left\langle(p \mid \widetilde{U})^{*} \circ\left(f_{1}, f_{2}\right)^{*}\left(\nu_{M}\right), o_{\tilde{g}}\right\rangle,
$$

which yields

$$
\omega(\varphi ; \tilde{g})=\left\langle\varphi^{*} \mu_{M}, o_{\tilde{g}}\right\rangle=\left\langle\left(f_{1}, f_{2}\right)^{*} \nu_{M}, o_{\tilde{g} \Gamma}\right\rangle=I\left(f_{1}, f_{2} ; \tilde{g} \Gamma\right)
$$

where $\nu_{M} \in H^{n}(M \times M, M \times M-\Delta M)$ is the Thom class, $o_{\tilde{g}}, o_{\tilde{g} \Gamma}$ are the fundamental homology classes around $\tilde{g}$ and $\tilde{g} \Gamma$, respectively, and $I\left(f_{1}, f_{2} ; \tilde{g} \Gamma\right)$ is the coincidence index of $f_{1}$ and $f_{2}$ at $\tilde{g} \Gamma$. $[13,15]$.

Lemma 3.2. Let $M$ be a compact connected nilmanifold. Suppose that $f, g: M$ $\rightarrow M$ are maps such that $L(f, g) \neq 0$. Then each (essential) coincidence class is of index +1 or -1 .

Proof. Following [8], there exist an orientable principal $T$-bundle $T \rightarrow M \rightarrow$ $N$, where $T$ is a torus and $N$ is a compact connected nilmanifold of dimension $\operatorname{dim} N<\operatorname{dim} M$, and fiber preserving maps $f^{\prime}, g^{\prime}$ such that

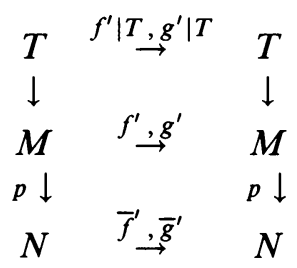

is commutative and $f \sim f^{\prime}, g \sim g^{\prime}$.

By a general position argument, $\bar{f}^{\prime} \sim \bar{\tau}$ and $\bar{g}^{\prime} \sim \bar{\eta}$ such that the coincidence set $C_{\bar{\tau}}, \bar{\eta}$ is finite. Then we can find maps $\tau, \eta: M \rightarrow M$ covering $\bar{\tau}, \bar{\eta}$ and $\tau \sim f^{\prime}, \eta \sim g^{\prime}$.

We may now assume without loss of generality that $f$ and $g$ are fiber preserving and $C_{\bar{f}, \bar{g}}$ is finite. Let $b \in C_{\bar{f}, \bar{g}}, T_{b}=p^{-1}(b)$, and $C_{b} \subset T_{b}$ be a coincidence class of $f \mid T_{b}$ and $g \mid T_{b}$. By [13, Theorem 3.3],

$$
L(f, g)=L\left(f\left|T_{b}, g\right| T_{b}\right) \cdot L(\bar{f}, \bar{g}) .
$$


Since $L(f, g) \neq 0$, both $L\left(f\left|T_{b}, g\right| T_{b}\right)$ and $L(\bar{f}, \bar{g})$ are nonzero. Since the fiber $T_{b}$ is a torus, it follows from [3, p. 125] that

$$
I\left(f\left|T_{b}, g\right| T_{b} ; C_{b}\right)= \pm 1 .
$$

Theorem 3.3 of [13] also gives the following local product formula:

$$
I\left(f, g ; C_{b}\right)=I\left(f\left|T_{b}, g\right| T_{b} ; C_{b}\right) \cdot I(\bar{f}, \bar{g} ; b) .
$$

Let $\alpha_{b}$ be the coincidence class of $\bar{f}$ and $\bar{g}$ containing $b$ and $\tilde{\alpha}$ be a coincidence class of $f$ and $g$ such that $p(\tilde{\alpha})=\alpha_{b}$. If $b^{\prime} \in \alpha_{b}$, then $I\left(f\left|T_{b^{\prime}}, g\right| T_{b^{\prime}} ; C_{b^{\prime}}\right)=I\left(f\left|T_{b}, g\right| T_{b} ; C_{b}\right)$ by 3.1 (see also [3, p. 125]). By summing the indices of all $b^{\prime} \in \alpha_{b}$ together with (IV) and (V), we obtain

$$
I(f, g ; \tilde{\alpha})= \pm I\left(\bar{f}, \bar{g} ; \alpha_{b}\right) .
$$

To complete the proof, we use induction on $m=\operatorname{dim} M$. For $m \leq 2$, the assertion follows from [3, p. 125]. Suppose that the assertion holds for dimension less than $m$. Since $\operatorname{dim} N<\operatorname{dim} M=m$, inductive hypothesis implies that $I\left(\bar{f}, \bar{g} ; \alpha_{b}\right)= \pm 1$ and hence, by (VI), $I(f, g ; \tilde{\alpha})= \pm 1$.

The following is a generalization of [1].

Theorem 3.3. Let $M$ be a compact connected nilmanifold. For any two maps $f, g: M \rightarrow M$,

$$
N(f, g)=|L(f, g)| \text {. }
$$

Proof. This theorem is due to R. Brooks [3] for $\operatorname{dim} M \leq 2$. For $\operatorname{dim} M \geq 3$, it follows from Lemmas 3.1 and 3.2 since $N(f, g)$ is just the number of essential coincidence classes each of which has the same index that is either +1 or -1 .

Corollary 3.4. Let $(E, p, B)$ be an orientable fibration where $F=p^{-1}(b) \quad(b \in$ $B)$ and $E$ and $B$ are compact connected nilmanifolds. For any given fiber preserving maps $f, g: E \rightarrow E$,

$$
N(f, g)=N\left(f_{b}, g_{b}\right) \cdot N(\bar{f}, \bar{g}) .
$$

Proof. This follows from the product formula (3.3) of [13] and Theorem 3.3

\section{REFERENCES}

1. D. Anosov, The Nielsen number of maps of nilmanifolds, Russian Math. Surveys 40 (1985), 149-150.

2. R. Brooks, Certain subgroups of the fundamental group and the number of roots of $f(x)=a$, Amer. J. Math. 95 (1973), 720-728.

3. Coincidences roots and fixed points, PhD Thesis, Univ. of California, Los Angeles, CA, 1967.

4. R. Brooks and R. Brown, A lower bound for the $\Delta$-Nielsen number, Trans. Amer. Math. Soc. 143 (1969), 555-564.

5. R. F. Brown, The Lefschetz fixed point theorem, Scott Foresman, IL, 1971.

6. A. Dold, Lectures on algebraic topology, Springer-Verlag, Heidelberg, 1972.

7. E. Fadell, Two vignettes in fixed point theory, Lecture Notes in Math., vol. 1411, SpringerVerlag, 1989, pp. 46-51.

8. E. Fadell and S. Husseini, On a theorem of Anosov on Nielsen numbers for nilmanifolds, Nonlin. Funct. Anal. Appl. 173 (1986), 47-53. 
9. J. Jezierski, The Nielsen number product formula for coincidences, Fund. Math. 134 (1989), 183-212.

10. B. Jiang, Lectures on Nielsen fixed point theory, Contemp. Math., vol. 14, Amer. Math. Soc., Providence, RI, 1982.

11. T. Kiang, The theory of fixed point classes, Springer-Verlag, Heidelberg, 1989.

12. C. McCord, Lefschetz and Nielsen coincidence numbers on nilmanifolds and solvmanifolds, Top. Appl., to appear.

13. M. Nakaoka, Coincidence Lefschetz numbers for a pair of fibre preserving maps, J. Math. Soc. Japan 32 (1980), 751-779.

14. H. Schirmer, Mindestzahlen von Koinzidenzpunkten, J. Reine Angew. Math. 194 (1955), 21-39.

15. J. Vick, Homology theory, Academic Press, New York, 1973.

Department of Mathematics, Bates College, Lewiston, Maine 04240

E-mail address: rbrooks@bat.bates.edu

E-mail address: pwong@bat.bates.edu 